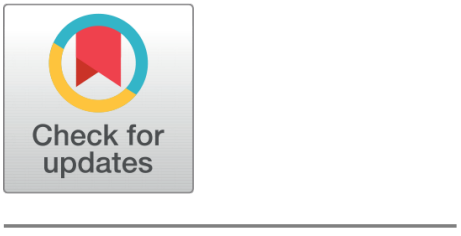

G OPEN ACCESS

Received: 19.10.2021

Accepted: 12.12 .2021

Published: 31.01 .2022

Citation: Preetha R, Vanaja S, Durga Devi K, Lathamanju R, Kurian NS, S JVJ (2022) Mass Sphering Approach for Three-Dimensional Reconstruction of Brain Tumor using MRI. Indian Journal of Science and Technology 15(3): 97-106. https://doi.org/ 10.17485/IJST/v15i3.1633

* Corresponding author.

preetharuban@gmail.com

Funding: None

Competing Interests: None

Copyright: ( 2022 Preetha et al. This is an open access article distributed under the terms of the Creative Commons Attribution License, which permits unrestricted use, distribution, and reproduction in any medium, provided the original author and source are credited.

Published By Indian Society for Education and Environment (iSee)

ISSN

Print: 0974-6846

Electronic: 0974-5645

\section{Mass Sphering Approach for Three-Dimensional Reconstruction of Brain Tumor using MRI}

\author{
R Preetha ${ }^{1 *}$, S Vanaja ${ }^{2}$, K Durga Devi ${ }^{1}, \mathrm{R}$ Lathamanju ${ }^{3}$, Nami Susan Kurian ${ }^{4}$, \\ Jacqulin Veda Jancy $\mathbf{S}^{\mathbf{1}}$ \\ 1 Assistant Professor (SG), Department of Electronics and Communication Engineering, SRM \\ Institute of Science \& Technology \\ 2 Assistant Professor (SG), Department of Electronics and Communication Engineering, \\ Easwari Engineering College, Chennai \\ 3 Associate Professor, Department of Electronics and Communication Engineering, SRM \\ Institute of Science \& Technology \\ 4 Assistant Professor, Department of Electronics and Communication Engineering, \\ Rajalakshmi Institute of Technology
}

\section{Abstract}

Objective: The objective of the research work is to reconstruct the brain tumor three-dimensionally with high degree of accuracy. Methods: This study describes 3D reconstruction of brain tumor using Mass Sphering Approach (MSA) algorithm. 39 weighted features are extracted from the non-tumor and tumor pixels. These weighted features are used to train the Support Vector Machine (SVM) algorithm. Number of training samples taken to train SVM algorithm are 268 and the testing sample are 64. The complete MR image set of a subject (64 axial slices) are detected for tumor pixels and these slices are concatenated to get volumetric tumor information. Findings: 5-step MSA algorithm is proposed which includes filtering, segmentation, classification, optimization and reconstruction. MR images are subjected to Rician noise which can be removed by a simple correction scheme, initiated to change the bias due to the Rician distribution of the noisy magnitude data. The filtered MR image slices are segmented and classified to detect the tumor areas and the tumor pixels are subjected for 3D reconstruction. The improvement in performance of MSA is depicted by comparing the algorithm with traditional SVM. Novelty: The accuracy achieved in detecting glioma and glioblastoma using MSA are $95.24 \%$ and $99 \%$ respectively which is highly remarkable.

Keywords: Glioma; voxel; Magnetic Resonance; Classification; Immune; Reconstruction

\section{Introduction}

Brain tumor detection is the most challenging task as the boundary of tumor is not confined and it may grow abnormally and cause damage to adjacent tissues ${ }^{(1)}$. A report from United News of India (UNI) reveals that, per year 28,142 new brain tumor cases 
were reported with a very high mortality rate of about 24,003 in the year $2018^{(2)}$. A survey is made to derive the characteristics of brain tumor and segmentation or classification techniques for successful detection of brain tumor ${ }^{(3)}$.

In the literature, a hybrid system of integrated $\mathrm{K}$-means clustering and Fuzzy $\mathrm{C}$ means model is developed for segmentation of tumor. The performance of this approach deals with minimizing the executing time with expected accuracy. Better accuracy is also reported in works which integrates K-means algorithm with improved support vector machine ${ }^{(4)}$. In theory, a new method called Quantum Artificial Immune System - deep spiking neural network (QAIS-DSNN) was proposed to segment and distinguish brain tumors from MR images ${ }^{(5)}$.

Many works have been reported recently, in brain tumor detection and classification using 'Support Vector Machine' (SVM) algorithm ${ }^{(6)}$. SVM is one of the traditional method for classification of tumor and non-tumor images which can be implemented with improvement for better accuracy. Therefore to improve the accuracy of SVM, it is integrated with immune algorithm and the brain tumor is three dimensionally reconstructed using simple MR images ${ }^{(7)}$.

The highly complex intra skull structures ${ }^{(8)}$, is developed to analyze the internal anatomy of the human body for efficient diagnoses and earlier detection of diseases. In this sequence, many techniques are introduced to reconstruct surfaces of internal human organs. Traditional surface rendering methods depend on objects characterized by polygonal surfaces, which define the 3D object. Fast marching method (FMM) prepares a comparative holographic projection for visual comparison between two abnormal follow-up magnetic resonance (MR) exams of glioblastoma patients to effectively visualize and assess tumor progression $^{(9)}$.

The above mentioned techniques are technically advanced and lead to complex calculations. To tackle this strenuous problem, Mass rendering techniques were developed to visualize information from the entire volume of data. The primary advantage of mass rendering techniques rather than surface rendering techniques is that mass rendering techniques retain added information in the final rendered image. In this paper, we focus on the visualization of the tumor based on the anatomical structure of the brain using an MR image set. This procedure is analogous to the computer graphics techniques used to render a 3D model of the tumor using the mass rendering algorithm.

In the remaining of this paper, materials and methods is reviewed in Section II; the results of algorithmic implementation are discussed in Section III; the experimental results and discussion are given in Section IV; and Section V concludes the paper.

\section{Material and Methods}

3D reconstruction has diverse applications in the medical field like complex intracranial structures ${ }^{(10)}$, craniofacial abnormalities ${ }^{(11)}$, microcalcifications in breast cancer ${ }^{(12)}$, artery visualization for cardiac applications ${ }^{(13)}$. Reconstruction of the tumor with its boundary completely depends on the accurate extraction of the irregularly shaped tumor from the corresponding MR images. The shape of the tumor can be extracted by segmenting the 3-D MR image. The complexity in visualizing abnormal cells in MR images can be overcome by reconstructing the tumor by using a volume rendering method.

Reconstruction is performed using MSA algorithm based on mass rendering. The mass sphering analysis presented in this section has five stages for reconstruction of brain tumor. Firstly, the entire MR image set of a single subject human brain is denoised and detached from the outer skull region using morphology and erosion methods. The image is de-noised for the purpose of enhancing the image quality which consecutively increases segmentation accuracy of MR images for more precise diagnosis. Several algorithms have been formulated for the effectiveness of image de-noising without losing the features of the image including spatial domain filtering methods, image transformation techniques and CNN based methods ${ }^{(14)}$. In MR images low signal intensities (Signal to Noise Ratio $(\mathrm{SNR})<2$ ) are consequently biased to noise which leads to blurring of significant details of images like edges and boundaries of tissue parts or structural details of an organ. A simple scheme is initiated to correct the bias due to the Rician distribution of the noisy magnitude data ${ }^{(15)}$. The additional noise in MR image is smoothed with the weights that are computed using pre-smoothed MR images.

The second stage in the process is segmentation and feature extraction. Segmentation of brain images accurately is a challenging area of research. Image segmentation plays a vital role in medical applications such as abnormality detection, quantitative analysis, and postsurgical assessment. This paper presents the conventional fuzzy c -means (FCM) clustering algorithm for the segmentation of brain MR images. Fuzzy c-means (FCM) algorithm plays an important role in image segmentation as it has better robustness and it can classify an image into different clusters using iterative method. The image is represented in various feature spaces, and the data points which has similar intensities, tend to group together depending on the distance from the pixel to the centroids in the feature domain. At the end of this stage, a segmented brain tumor with its exact boundary is achieved. From this segmented image, 39 features are extracted in order to classify whether the MR image has tumor or not. 
The third stage in the process is to classify the tumor and non-tumor images. This includes two phases namely training and testing phase. In the training phase, the SVM algorithm is trained with MR images including tumor and non-tumor. In testing phase, the SVM algorithm classifies the given test images based on the features trained in it. Number of training samples taken to train SVM algorithm is 268 (including tumor slices and 121 abnormal brain slices) and the testing sample is 64.

The fourth stage is to apply an iterative immune algorithm to classify tissue substances repeatedly by using the previous classified results as a new set of training samples for the next iteration to improve the accuracy and sensitivity. These above mentioned steps are done for 64 axial slices of MR images obtained from a single patient. At the final stage these segmented tumor slices are concatenated to get a volumetric 3D tumor.

The fifth and the final stage is to concatenate the tumor slices using MR image slice deformation algorithm to view the 3D volumetric data at different angles. Image interpolation is an important mechanism for establishing new points of data inside a specific domain of a discrete set of known points of data. Image interpolation is used to eliminate such artifacts and crocks.

\section{MSA}

A detailed step-by-step implementation of the MSA is shown in Fig.1 and is described as follows.

1. Firstly, a database is created by denoising and removing the outer skull region of brain MR image using Rician filtering and morphology methods respectively.

2. The contrast of the MR images depends on the attenuation of the structures being imaged. There are three different sequences in contrast namely T1 weighted (T1), T2 weighted (T2) and Proton density (PD). The Region of interest is selected from $M R$ slices $\mathrm{MR}_{i}=(\mathrm{T} 1, \mathrm{~T} 2, \mathrm{PD})$ using Fuzzy C-means algorithm followed by weighted feature extraction of the tumor pixels $\mathrm{FW}_{\text {init }}(\mathrm{T} 1, \mathrm{~T} 2, \mathrm{PD})$.

3. An intial training set is developed for classifying the testing samples using SVM denoted by FWSVM init $_{\text {. }}$

4. SVM algorithm is further enhanced by tuning the parameters using Immune algorithm represented as FWI -SVM init .

5. 3D reconstruction of Feature weighted Immune SVM slices is constructed by concatenating and interpolating the samples.

\subsection{Image acquisition}

MR image sequences were used to visualize different anatomical structures of the brain. The initial task is to acquire the MR image from the radiologist and to detach the outer skeletal region from the brain anatomy. MR images of T1 axial slices were acquired on a head scanner using an inversion recovery sequence $(\mathrm{TE}=105.0, \mathrm{TR}=9000)$. Twelve subject images each having approximately Fifty one images was acquired from the radiologist with a slice thickness of $5.0 \mathrm{~mm}$. The input to the system is the whole set of 3D MR image slices of a single patient.

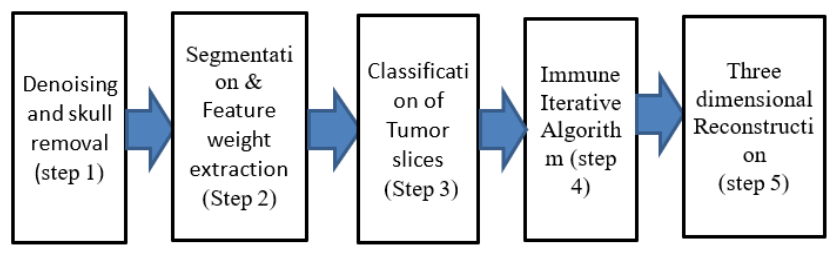

Fig 1. Flowchart of MSA Approach

\subsection{Denoising}

From the survey done, it is known that in noisy backgrounds the intensity in the MR image is governed by rice distribution. The most direct way to acquire a desirable result is to denoise the MR image before segmentation. Separating Rician noise from MR image is difficult, as it is signal dependent. A correction scheme must be introduced to reduce the bias before subjecting the image for clustering. Correlated Rician noise removal techniques are used to denoise the MR image as it is corrupted by Rician distributed noise and suffers from contrast related problems. This is a two-step denoising procedure in which the bias is isolated from the squared magnitude image and the denoising is performed on the square root of the image in the wavelet domain. The results of filtering are shown inFigure 2The obtained Rician filtered MR data deserves a further segmentation process. The pixels of the skeletal region usually take the form of isolated bright areas in the brain anatomy. To eliminate this morphological filter including dilation, erosion operations are used as the post processing technique to remove them. 


\subsection{Segmentation}

In order to diagnose the malformations in the MR images, image segmentation is the challenging and complex method. Segmentation also plays a vital role in visualizing the anatomy of human tissue. It is noteworthy that in a human brain MR image, the borders of tumor tissue are extremely irregular. In medical image segmentation, multiple deformable methods and region based algorithms are used to locate the frontier of the tumor. But the major limitations in these algorithms are: (a) non-linear distribution of real data (b) user interaction (c) poor convergence.

The most promising clustering technique in the field of brain tumor detection is by fuzzy C means (FCM), which is one of the most effective and robust algorithms for tumor detection. Clustering is called the process of segmenting a group of objects into a number of homogeneous clusters. Fuzzy $\mathrm{C}$ method is extensively chosen as the data component belongs to more than one cluster and each component has a set of membership levels. The goal of this algorithm is on diminishing the objective function. The objective function is taken into consideration based on the membership value and cluster centers. Even though the method has high computational complexity, it outperforms other methods in efficiency and convergence rate. Figure 2 shows the results of segmentation.
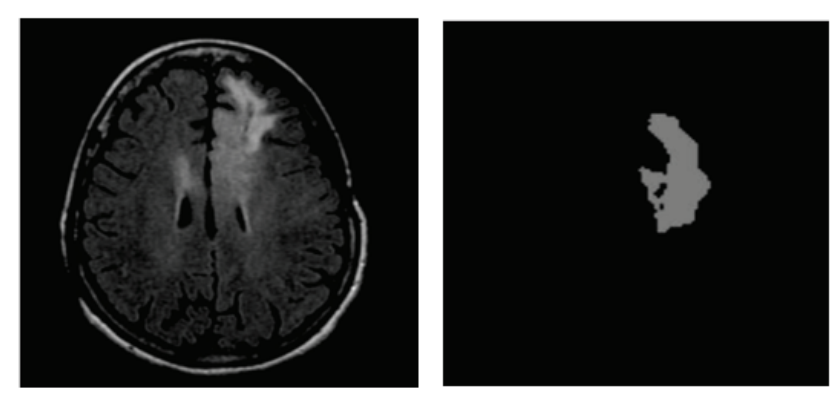

Fig 2. a) Original raw image obtained from MRI scanner b) Tumor Image segmented from2a after FCM

\subsection{Weighted Feature extraction}

Feature extraction is a method of constructing quantitative information from the original image. The features are based on color, texture, contrast and shape. Instead of complete image, the features represents the key information of the image which helps in reducing the dimensionality factor. Almost 39 features are extracted for the work, which stands as the base factor, to classify the normal and abnormal tumors. The weighted features are obtained from averaging every features in all four orientations $\left(0^{\circ}\right.$, $\left.45^{\circ}, 90^{\circ}, 135^{\circ}\right)$, in order to improve the accuracy.

\subsection{Feature weighted immune classification}

Machine learning methods for automatic brain tumor detection is explored to proceed with diagnosing the boundary of tumor cells. Introduction of such machine learning algorithm has made the work easy for the radiologists and experts in medical field. These algorithms deals with patterns and complex data more accurately. Machine learning can be classified into supervised and unsupervised algorithm. SVM is a supervised machine learning algorithm that can solve both linear and non-linear binary classification problems. For $n$-dimensional space, the input data belongs to class 1 or class 2 and the labels are -1 and +1 respectively. The features extracted from the original image is the input of SVM. Constructing maximal margin hyperplane is the goal of SVM to classify the input data. The SVM tries to maximize the distance between the features that are nearer to the separation plane. For the training phase of the SVM classifier, one slice, which was the ground truth (GT) of the tumor, was randomly chosen in each subject dataset. Accuracy, sensitivity, and specificity factors are determined. Amongst the different kernels like sigmoidal, polynomial, linear, and radial basis function (RBF), by literature, RBF is selected for the optimum separation between the two classes, as it gives good classification. To obtain optima and to improve accuracy, an iterative immune algorithm is used to classify tissues repeatedly using the results of previous classification.

\section{Algorithm 1}

SVM algorithm for tumor detection in brain image

1. In binary classification, let the training dataset be as $\mathrm{S}$ with $\mathrm{x}$ and $\mathrm{y}$ labeled samples. $\mathrm{S}=(\mathrm{x} 1, \mathrm{y} 1), \ldots . .,(\mathrm{xl}, \mathrm{yl})$, where $\mathrm{xi}$ e $\mathrm{Rd}$ and the class label yi e $\{1,-1\}$. 
2. The training algorithm is simplified by transforming quadrature programming problem to linear problem using,

$$
\min _{w, b, \varepsilon i} \frac{1}{2}\|w\| 2+C \sum_{i=1}^{n} \varepsilon i
$$

Such that $y \mathrm{i}(w \cdot x \mathrm{i}-b) \geq 1-\varepsilon \mathrm{i}, 1 \leq i \leq l$, Where $\mathrm{C}$ is the regularization parameter, $\varepsilon \mathrm{i}$ is the slack variable, $\mathrm{W}$ is the normal vector to the hyper plane and $\mathrm{b}$ is the bias applied.

3. The lagrangian multiplier is introduced $\alpha \mathrm{i} \geq 0$ and the quadratic problem is given by

$\max \sum_{\mathrm{i}=1}^{\mathrm{l}} \alpha \mathrm{i}-\frac{1}{2} \sum_{\mathrm{i}} \sum_{\mathrm{j}} \alpha_{\mathrm{i}} \alpha_{\mathrm{j}} \mathrm{y}_{\mathrm{i}} \mathrm{y}_{\mathrm{j}} \mathrm{x}_{\mathrm{i}}^{\mathrm{T}} \mathrm{x}_{\mathrm{j}}$

4. The decision function is given by

$$
f(x)=\operatorname{sgn}(w \cdot x+b)
$$

5. Repeat step 2 to 4 until the whole process is completed

6. End

\section{Algorithm 2}

Immune SVM to optimize the parameters of $\mathrm{C}$ and $\gamma$

1. $\mathrm{N}$ potential solutions from the solution space are randomly selected as the initial Antibodies $(\mathrm{Ab})$

Set $1=(C 1, \gamma 1) k$, Set $2=(C 2, \gamma 2) k, \ldots$. Set $N=(C m, \gamma m) k$

2. $\quad$ Si $j=1-\frac{\left\|A b_{i}-A b_{j}\right\|}{\max _{1 \leq i, j \leq N}\left\|A b_{i}-A b_{j}\right\|}, \quad \mathrm{i}, \mathrm{j}=1, \ldots, \mathrm{N}, \mathrm{i} \neq \mathrm{j}$

3. Based on the similarity, Abs with high similarities are suppressed. From the population of $\mathrm{N}=20,5$ similar antibodies are removed and the remaining 15 antibodies are given as the input to the next generation

$$
\mathrm{Ab}_{\mathrm{i}}^{*}=\mathrm{Ab}_{\mathrm{i}}-\left(1-\mathrm{e}\left\|\mathrm{A} \mathrm{b}_{\mathrm{i}}-\mathrm{Ag}\right\|\right)\left\|\mathrm{Ab} \mathrm{b}_{\mathrm{i}}-\mathrm{Ag}\right\|
$$

4. Repeat step 1 to 3 until the parameters are optimized

5. End

\subsection{MRI slice deformation technique}

The segmented tumor sections are concatenated and interpolated, and the $3 \mathrm{D}$ algorithm relies on a factorization that simplifies projection from volume to image. It produces an object-order algorithm with the comparable benefits of the factorization of an image-scan order algorithm called the MR image slice deformation technique. The different views of the volume of data can be viewed by, rotating the voxels of the volume to the right side before the projection of the 3Dimensional information into a 2Dimensional -plane. The projection process is complicated as the random positions of samples are rotated. The MR image slice deformation simplifies this projection by first tilting the object planes, then projecting to another plane and twisting the viewed points (i.e. voxels of the data).

\section{Results and Discussion}

Simple conventional SVM is trained and tested with the datasets which shows the tumor detection accuracy of about $88 \%$. The same dataset is also tested with the proposed MSA and the accuracy steadily rises to 95\%. MSA algorithm is an improved SVM which includes feature weighted methodology and immune algorithm (FWISVM) in it. The proposed MSA outperforms SVM in sensitivity and specificity as well. The comparison of the SVM and the proposed MSA is discussed below.

In this study, a large dataset with three different types of T1 weighted tumor MR images with a 256x256 matrix and 5mm slice thickness were acquired from the radiologists. The datasets have tumors like Glioblastoma and Lower Grade Glioma. The images are denoised and clustered for tumor segmentation. The work implemented is compared with traditional SVM method.

\subsection{Segmentation Efficiency}

Segmentation efficiency is the ratio of true positive value to the ground truth tumor given by SE= (True positive/Ground truth tumor) ${ }^{\star} 100$. Table 1 figure out the segmentation efficiency of the glioma at its lower and higher grade using our algorithm. Also, this proposed method shows better results for glioblastoma than lower grade glioma and meningioma. The clustering method used here performs well on a good contrast image but its performance deteriorates if the image quality decreases. 
Table 1. Comparison of Performance based on e fficiency

\begin{tabular}{cl}
\hline Type of Tumor & Segmentation Efficiency \\
\hline Lower grade glioma & 94.39 \\
Glionghioblastoma & 96.75 \\
\hline
\end{tabular}

\subsection{Comparison of Performance Measure Using SVM with MSA}

Computer aided methods like image processing and machine learning to help in identifying suspicious areas of the brain. Machine learning has two phases, namely the training and the testing phase. The total dataset given for the training phase is 147 tumor slices and 121 abnormal brain slices. The algorithm is implemented in MATLAB on a PC with 3GHZ and 1.93GB RAM. The subject images are implemented with both SVM and FWISVM algorithm and the results are observed. The comparison graph shows that the FWISVM outperforms SVM in all the 3 subject cases. The parameters taken into consideration for performance evaluation are True Positive (TP), True Negative (TN), Sensitivity (Sn), Specificity (Sp), and Accuracy (Ac). Sensitivity is the test to identify the tumor correctly, whereas the specificity is the test to identify a non-tumor correctly. Accuracy is the test that differentiates tumor and non-tumor correctly. The subject wise result is evaluated for SVM and FWISVM. The number of cases aright identified as patient is termed as True Positive. The number of cases aright identified as healthy is termed as True Negative. The ability to find out the patient cases correctly is termed as sensitivity. Sensitivity can be estimated from the proportion of true positive in patient cases. Mathematically, sensitivity can be calculated by,

Sensitivity $=(\mathrm{TN}+\mathrm{TP}) /(\mathrm{TN}+\mathrm{TP}+\mathrm{FN}+\mathrm{FP})(8)$

The ability to find out the healthy cases correctly is called specificity. Specificity can be estimated from the proportion of true negative in healthy cases. Mathematically, sensitivity can be calculated by,

Specificity $=$ TN $/(\mathrm{TN}+\mathrm{FP})(9)$

Accuracy is the measure of differentiating the patient and healthy cases correctly. Accuracy can be estimated from the proportion of TP and TN in all evaluated cases. Mathematically, accuracy can be calculated by,

Accuracy $=(\mathrm{TN}+\mathrm{TP}) /(\mathrm{TN}+\mathrm{TP}+\mathrm{FN}+\mathrm{FP})(10)$

When accuracy of all 3 subjects is compared with the two different algorithms, FWISVM outperforms the existing SVM in all the 3 cases. The result shows that the FWISVM gives the highest overall accuracy.

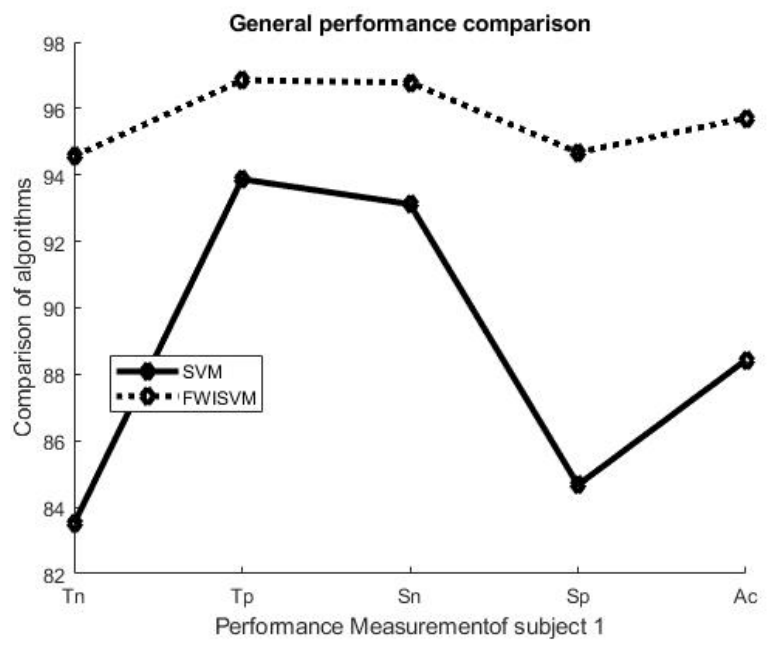

Fig 3. Comparison of SVM and MSA (FWISVM) algorithm including all MRI slices of brain for subject 1

Fig. 3 clearly depicts that Sensitivity, Specificity and accuracy are increased for FWISVM when compared with SVM. One of the most important objectives of FWISVM is to increase the TP values. Drastic increase is observed in Tp value for the proposed algorithm than the conventional. There is a high increase in the values of Tp, Tn, Sn, Sp and Ac from $80 \%$ to around $95 \%$ when estimated with FWISVM.

Taking subject 2 into consideration, noted in Figure 4, 95\% is still maintained by the proposed algorithm for all the parameters and the key observation in this graph is the specificity factor. Its value is raised from $75 \%$ to $94 \%$ by using the 


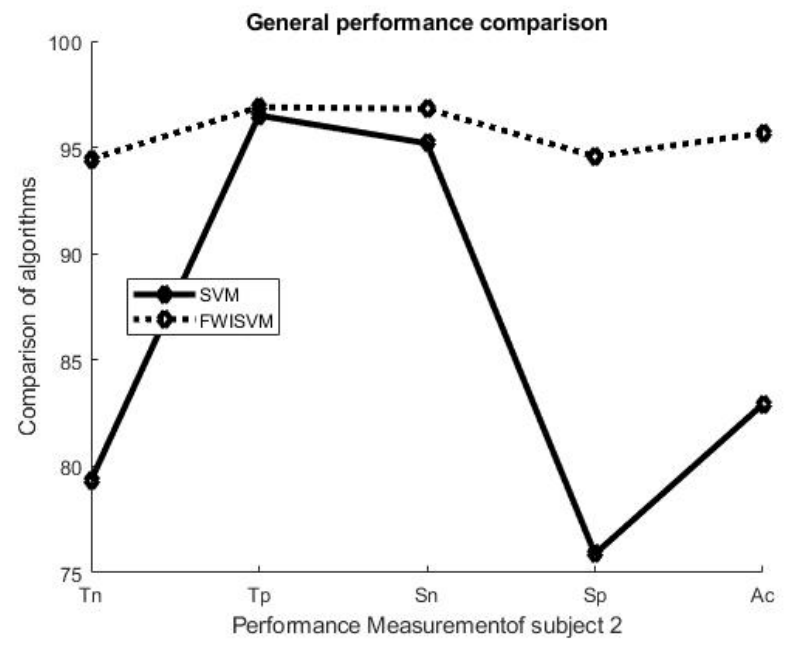

Fig 4. Comparison of SVM and MSA (FWISVM) algorithm including all MRI slices of brain for subject 2

proposed algorithm. From Fig.5 it can be inferred that IFWSVM outperforms SVM by retaining all the parameter values in the range of $95 \%$ making SVM less efficient to be used in real time.

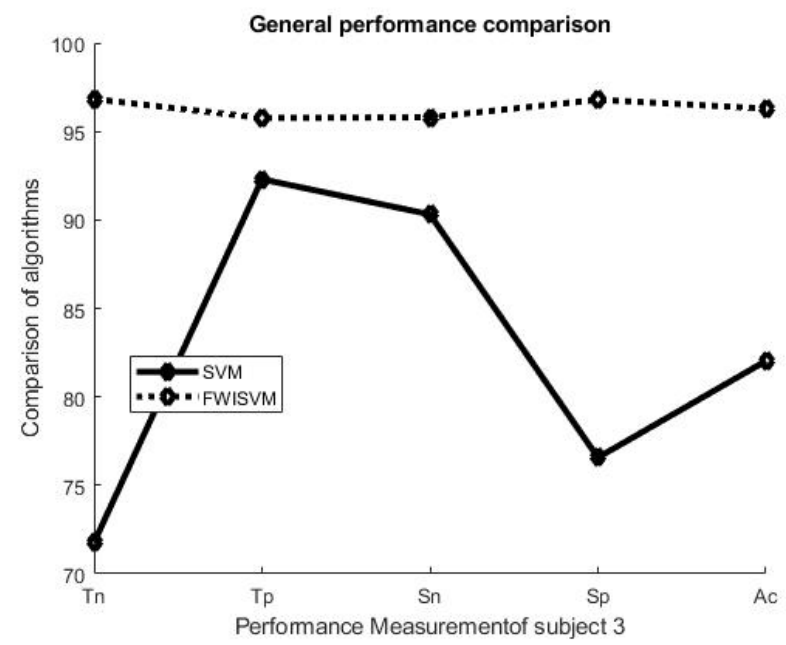

Fig 5. Comparison of SVM and MSA (FWISVM) algorithm including all MRI slices of brain for subject 3

The training time taken by the algorithm for glioblastoma is 420.99 s. However, it is practical and tolerable. Table 2 depicts the performance measure of the classification algorithm for the abnormal region of the brain.

Table 2. General performance measure for glioma using iterative SVM

\begin{tabular}{lll}
\hline & \multicolumn{2}{c}{ Glioma } \\
\cline { 2 - 3 } & Lower Grade Glioma & Glioblastoma \\
\hline $\mathrm{TP}(\%)$ & 91.56 & 93.85 \\
$\mathrm{TN}(\%)$ & 95.85 & 98.28 \\
Sensitivity (\%) & 92.67 & 97.5 \\
Specificity (\%) & 94.45 & 96.5 \\
Accuracy (\%) & 95.24 & 99 \\
\hline
\end{tabular}


The table indicates that the accuracy value obtained for glioblastoma is higher than lower grade glioma.

\subsection{Three-dimensional reconstruction}

MSA algorithm was implemented in MATLAB on a PC with 3GHZ and 1.93GB RAM system and it has been tested on real MR data obtained from the database. An example of the MRI axial slices of a subject is shown in Fig. 6.

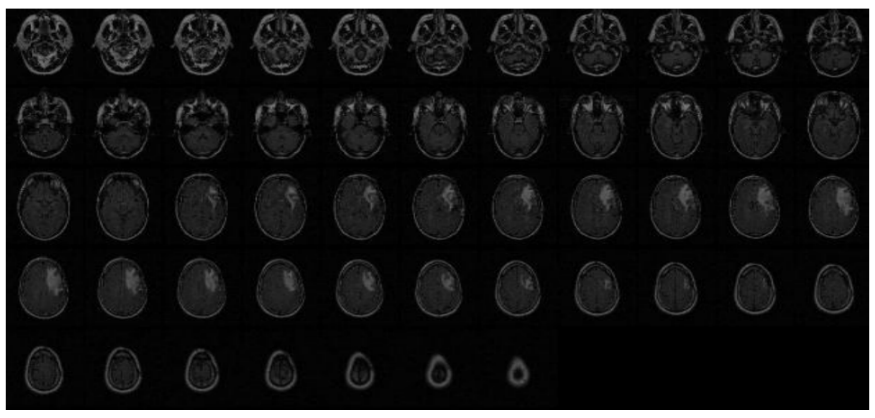

Fig 6. MRI axial sets of glioma of a single patient

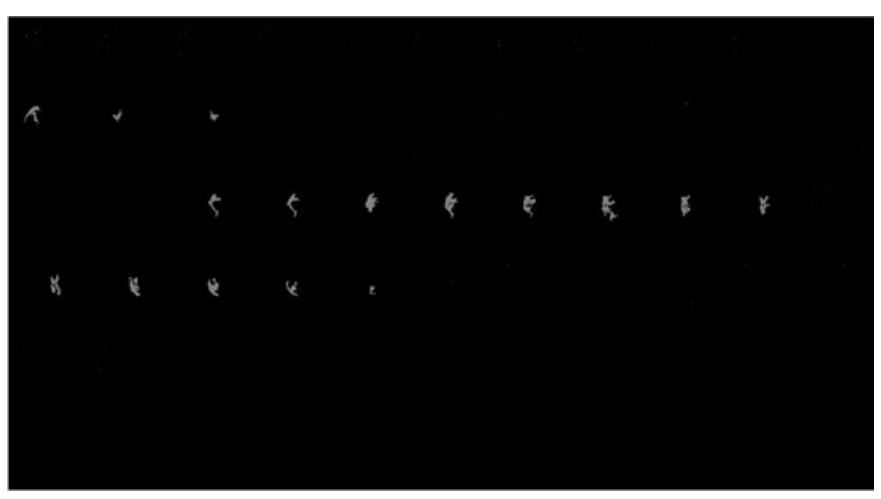

Fig 7. Segmented slices from the dataset

In this section, we present the application of the MSA approach for 3-D brain images. Before applying any clustering algorithm, extra cranial layers were removed from all images and a semi-automated technique is performed for the real MR data. Fig. 5 shows the results of applying FCM on an MR brain image that has 1-mm cubic voxels and $40 \%$ image intensity inhomogeneity. The three tissue classes in the brain anatomy are gray matter (GM), white matter (WM), and cerebrospinal fluid (CSF) where background pixels are ignored.

The axial MRI dataset from a subject is segmented and it is shown in Figure 7. As the original 3D T1- weighted MR image was anisotropic, the resolution in the axial direction is lower than that of the other two directions. Due to this anisotropy, 3D reconstruction of the brain tissue often had a strong stair effect. To eliminate this effect and to improve the resolution, Rician filtering and clustering is used, which makes the image isotropic. We applied our MSA to T1 weighted MRI head scans to three-dimensionally reconstruct the brain tumor accurately. In order to improve visual perception, MRI Slice Deformation was employed to generate Figure 8, which is helpful for neurosurgeons to learn about and locate the brain tumor accurately.

The quality of the reconstructed results is measured using two factors for three different patients, namely noise variance (NV) and contrast to noise ratio (CNR). Noise variance determines the image quality by measuring the discrepancy between heterogeneous regions of brain anatomy. CNR is a measure of quality of the image based on contrast and it provides knowledge to detect induced fluctuation. The parameters can be obtained using the following equations.

$N V=10^{\wedge}(\mathrm{SNR} / 10)$

$$
\mathrm{CNR}=20 \log 10(\mathrm{SNR})
$$

Where SNR is Signal to Noise ratio.

The results are tabulated in Table 3. 

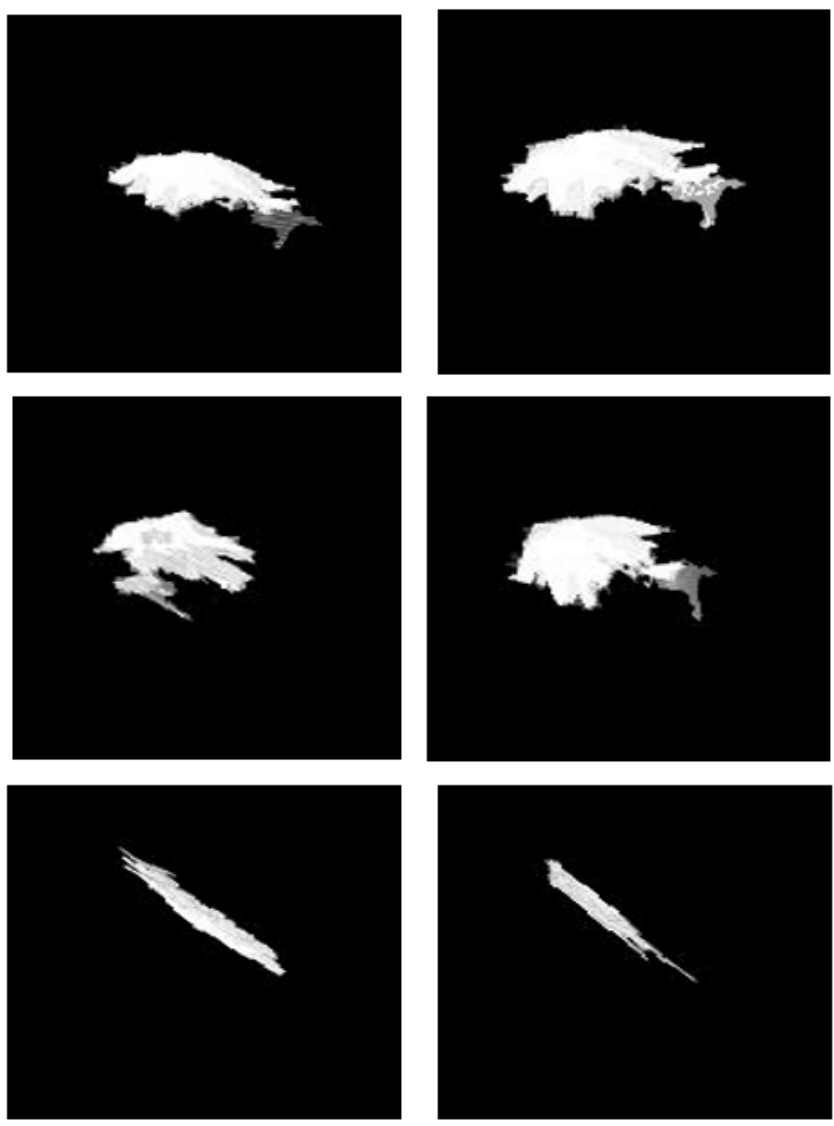

Fig 8. Three-dimensionally rendered view of the internal structures of the tumor along the mid axial plane through the MSA approach in $0^{\circ}$, $45^{\circ}, 60^{\circ}, 90^{\circ}, 120^{\circ}$ and $240^{\circ}$ inclination respectively.

Table 3. Quality factors measured for the THREE-DIMENSIONAL tumor image

\begin{tabular}{llll}
\hline Image Quality/subject & Patient $\mathbf{1}$ & Patient 2 & Patient 3 \\
\hline Noise variance & $1.13 \mathrm{E}-08$ & $3.66 \mathrm{E}-08$ & $1.81 \mathrm{E}-08$ \\
$\mathrm{CNR}$ & 2.41135 & 2.11232 & 3.04457 \\
\hline
\end{tabular}

\section{Conclusion}

This study presents a new approach, called MSA to deal with 3d brain tumor visualization. In this work, a 3D image reconstruction method is proposed based on MSA and is implemented for brain tissue reconstruction. In order to avoid human supervision, a small set of ground truth tumors is selected as training samples based on anatomical structures as a primary training set to ensure that all the required tumor tissues are included in a region of interest. The Feature weighted Immune SVM is further used to refine the initial set of training samples through a support vector machine for precise classification. Experimental results demonstrate that the proposed MSA can perform on the entire image cube and the overall accuracy obtained is $92 \%$. We conclude that image segmentation and reconstruction of brain tissue for $3 \mathrm{D}$ T1-weighted MRI is feasible, through which we can improve the cognitive ability of neurosurgeons to perform neurosurgical planning. The future work is to implement the novel proposed algorithm in real time images.

\section{References}

1) Amin J, Sharif M, Haldorai A. Brain tumor detection and classification using machine learning: a comprehensive survey. Complex \& Intelligent Systems. 2021. doi:10.1007/s40747-021-00563-y.

2) Tiwari A, Srivastava S, Pant M. Brain tumor segmentation and classification from magnetic resonance images: Review of selected methods from. Pattern Recognition Letters. 2014;131. doi:10.1016/j.patrec.2019.11.020. 
3) Biratu ES, Schwenker F, Ayano YM, Debelee TG. A Survey of Brain Tumor Segmentation and Classification Algorithms. Journal of Imaging. 2021;7(9):179179. doi:10.3390/jimaging7090179.

4) Cao J, Wang M, Li Y, Zhang Q. Improved support vector machine classification algorithm based on adaptive feature weight updating in the Hadoop cluster environment. PLOS ONE. 2019;14(4):e0215136-e0215136. doi:10.1371/journal.pone.0215136.

5) Ahmadi M, Sharifi A, Hassantabar S, Enayati S. QAIS-DSNN: Tumor Area Segmentation of MRI Image with Optimized Quantum Matched-Filter Technique and Deep Spiking Neural Network. BioMed Research International. 2021;2021:16-16. doi:10.1155/2021/6653879.

6) Nalepa J, Kawulok M. Selecting training sets for support vector machines: a review. Artificial Intelligence Review. 2019;52:857-900. Available from: https://doi.org/10.1007/s10462-017-9611-1.

7) Guo L, Li Y, Miao D, Zhao L, Yan W, Shen X. 3-D Reconstruction of Encephalic Tissue in MR Images Using Immune Sphere-Shaped SVMs. IEEE Transactions on Magnetics. 2011;47(5):870-873. doi:10.1109/tmag.2010.2072776.

8) Edward JF, Zappulla R, Yang WC. Color 3-D Imaging of Normal and Pathologic Intracranial Structures. Computer Graphics and Applications IEEE. 1984;(4):9-14. Available from: https://ieeexplore.ieee.org/abstract/document/4055913.

9) Abdelazeem RM, Youssef D, El-Azab J, Hassab-Elnaby S, Agour M. Three-dimensional visualization of brain tumor progression based accurate segmentation via comparative holographic projection. PLOS ONE. 2020;15(7). Available from: https://doi.org/10.1371/journal.pone.0236835.

10) Fawzi A, Achuthan A, Belaton B. Brain Image Segmentation in Recent Years: A Narrative Review. Brain Sciences. 2021;11(8):1055-1055. doi:10.3390/brainsci11081055.

11) Clark AE, Biffi B, Sivera R, Dall'Asta A, Fessey F, Wong TL, et al. Developing and testing an algorithm for automatic segmentation of the fetal face from three-dimensional ultrasound images. Royal Society Open Science. 2020;7(11):201342-201342. doi:10.1098/rsos.201342.

12) Chaudhury S, Rakhra M, Memon N, Sau K, Ayana MT. Breast Cancer Calcifications: Identification Using a Novel Segmentation Approach. Computational and Mathematical Methods in Medicine. 2021;2021:9905808-9905808. doi:10.1155/2021/9905808.

13) Chen C, Chen Q, Huaqi Q, Giacomo T, Jinming D, Wenjia B, et al. Deep Learning for Cardiac Image Segmentation: A Review. Frontiers in Cardiovascular Medicine. 2020;7. Available from: https://doi.org/10.3389/fcvm.2020.00025.

14) Fan L, Zhang F, Fan H, Zhang C. Brief review of image denoising techniques. Visual Computing for Industry, Biomedicine, and Art. $2019 ; 2(1): 7-7$. doi:10.1186/s42492-019-0016-7.

15) Pal C, Das P, Chakrabarti A, Ghosh R. Rician noise removal in magnitude MRI images using efficient anisotropic diffusion filtering. International Journal of Imaging Systems and Technology. 2017;27(3):248-264. doi:10.1002/ima.22230. 